Idalith León *

\title{
AMBIENTES DE APRENDIZAJE HIPERMEDIALES: Categorizacion y posibilidades pedagógicas
}

\section{A. Tecnologías de la información y la comunicación: las ayudas educativas del nuevo milenio}

El análisis de lo educativo ha llevado a identificar empíricamente dos modalidades con las cuales se pretende aproximar la conducta de los individuos de un grupo a los valores y normas conformados en un modelo del grupo social de referencia: las espontáneas y las formales. Las primeras no han sido intencionalmente desarrolladas; las segundas, se han producido de manera intencional con ciertos propósitos, mediante procesos racionalizados y requieren generalmente de un soporte administrativo para funcionar, la enseñanza escolar puede catalogarse como una es trategia pedagógica de las modalidades formales.

Ahora bien, en cualquier modelo de enseñanza-aprendizaje de las modalidades formales, es factible identificar por lo menos 4 variables: el agente, el sujeto, el objeto y el medio ambiente. El siguiente esquema (Legandre, 1996) representa la manera como podrían interpretarse las relaciones entre estas variables.

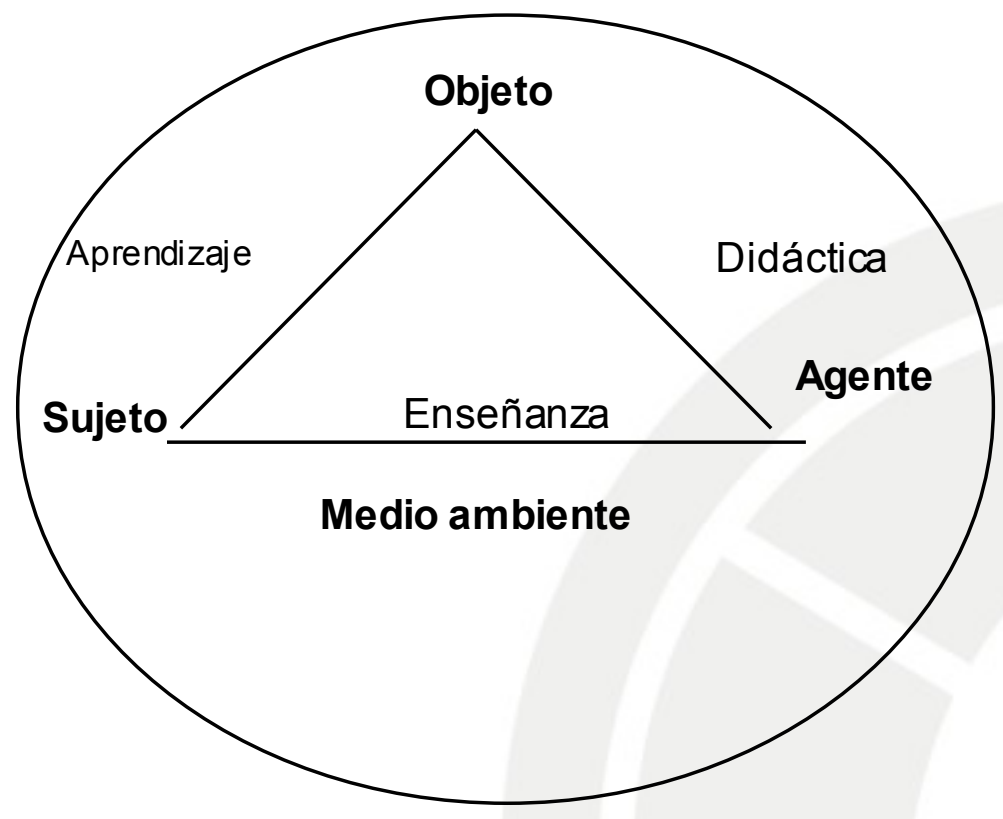

\footnotetext{
* Mg. Lingüística hispánica Instituto Caro y Cuervo. Mg. Tecnologías de la Información Aplicadas a la Educación Universidad Pedagógica Nacional. Coordinadora proyecto Ambientes computarizados para el Aprendizaje de las Ciencias Humanas Facultad de Humanidades. Universidad Pedagógica Nacional.
} 
Interesa ahora centrarse en la variable medio ambiente y dentro de ella en las llamadas ayudas educativas, como elemento presente desde tiempos inmemoriales en cualquier proceso de enseñanza-aprendizaje de las modalidades formales. En efecto, bien sea bajo la forma de una roca, de una pizarra, de un papiro, de un tablero, las ayudas educativas han estado acompañando permanentemente la labor del maes tro (agente) y mediatizando su interacción con el estudiante(sujeto), para bien o para mal y su evolución, al menos en cuanto a su deber ser, bien podría medirse de acuerdo a la evolución de la tecnología, que de hecho se hace presente en la educación, entre otras manifestaciones, a través de artefactos: el libro de texto, la radio, el televisor y ahora las tecnologías de la información y la comunicación han irrumpido en los ambientes educativos dando lugar a transformaciones que en muchos casos se quedaron en el plano de la novedad, sin convertirse en verdaderas revoluciones generadoras de prácticas pedagógicas innovadoras. ¿Pasará lo mismo con la nueva generación de artefactos producidos por la tecnología microelectrónica e informática?

Así, llegando al momento histórico que compartimos es impensable desligar las que de manera legítima pueden seguirse llamando ayudas educativas, de las tecnologías de la información y la comunicación (TICs) cuya incursión es evidente en todas las esferas de la actividad humana, dentro de ellas la educación. De ahí que se haga indispensable una sucinta revisión de la incorporación de la informática a la educación, para luego establecer los fundamentos pedagógicos que subyacen al uso de esta nueva generación de ayudas educativas.

La idea de vincular el computador a la educación surge del uso de la televisión como herramienta pedagógica. Su inclusión en el ámbito educativo se puede ubicar entre los años 60s y 70s, con el uso del computador en la enseñanza básica de las Matemáticas. Aunque en este período empiezan a ingresar los computadores a la escuela, existe una falta de comprensión entre los profesores acerca de lo que representan en el pensamiento y la forma como influyen en el proceso de aprendizaje. Muchos de los profesores pueden estar de acuerdo en considerar el computador como una herramienta inteligente, tanto para el maestro como para el alumno, pero todo dependerá de la capacidad del propio profesor para aprovechar los recursos potenciales del ordenador.

El uso de los micro-computadores en procesos educativos ha sido abordado desde diferentes perspectivas, que han estado influidas tanto por concepciones pedagógicas como por las posibilidades tecnológicas de cada época. Así, los desarrollos computarizados con fines educativos pueden agruparse en las siguientes categorías: 


\section{Instrucción Asistida por Computador (CAl)}

Sus antecedentes más directos pueden ser la máquina de enseñar de Skinner y antes la de Pressey, en 1926. Se basa en la enseñanza programada, en la que el material de estudio se presenta en el monitor, el estudiante introduce su respuesta mediante el teclado, la computadora la compara con la que podría considerarse correcta y según el resultado puede volver a plantear el ejercicio hasta que el estudiante introduzca la respues ta correcta, o indicarle qué tipo de error cometió, o continuar con el programa si la respuesta es acertada.

Esta línea de uso, que plantea básicamente ejercicios repetitivos y de práctica, aún continúa utilizándose en casos muy específicos, en los que se necesita entrenamiento rápido y eficaz. Tal es el caso de la enseñanza y capacitación para adultos, la educación especial y la enseñanza de conocimientos es pecializados.

\section{Los Micromundos}

Este enfoque de Seymour Papert fue diseñado, no sólo para promover formas de pensamiento a través del juego con el computador y de la creación de ambientes de aprendizaje, sino para promover además, la apreciación de ideas formales y de conceptos. Muestra un compromiso con modelos de aprendizaje cognitivos y de desarrollo (Piaget) que inspiran el aprendizaje por exploración y que se interesan por la interpretación y explicación de las relaciones del individuo con su mundo exterior.

Aquí se inscribe el proyecto LOGO que mediante las acciones que puede ejecutar una tortuga, le permite al niño visualizar conceptos abstractos y dar instrucciones que corresponden a acciones que podría realizar con objetos reales. Estos procesos pueden ayudar al niño a desempeñarse mejor en la etapa de las operaciones concretas. Pero de igual manera LOGO puede utilizarse como un ambiente de desarrollo de habilidades correspondientes a la etapa de operaciones formales, al introducir al niño en el mundo de la programación, que siendo relativamente sencilla, propicio la aplicación del pensamiento hipotético-deductivo y de las relaciones lógicas.

\section{Los Sistemas Inteligentes}

Desde la década de los 50 los avances de la Inteligencia Artificial empezaron a incursionar en el mundo de la tecnología educativa dando lugar a desarrollos mucho más sofisticados y potentes, como los Sistemas Expertos y los Tutores Inteligentes.

Aunque es innegable el valor y la ayuda que estos sistemas inteligentes representan para los procesos de enseñanza y de aprendizaje, no deben 
desconocerse sus limitaciones que están referidas básicamente a factores de tiempo, dinero y recursos humanos.

Las principales características de un sistema inteligente son el alto grado de interactividad y la capacidad de adaptarse al usuario. Dentro de este grupo se encuentran diversas modalidades, como los sistemas expertos, las simulaciones y los tutores inteligentes. Estos últimos nacen explícitamente con un propósito educativo y podrían considerarse como los que ofrecen mayor número de posibilidades pedagógicas, ya que constan de 4 módulos, cada uno con funciones específicas: la base del conocimiento, que almacena el saber; el motor de inferencia, en el que se explicitan mediante reglas las relaciones entre conceptos, teniendo como modelo un experto; el módulo de modelación del estudiante, que es el encargado de acomodar el sistema a las características del usuario y la interface, que permite la interacción hombre-máquina.

En cuanto a las simulaciones, también son programas muy potentes que, aunque tienen aplicaciones en muy diversos campos, han sido utilizadas con excelentes resultados en procesos educativos. Una simulación es un programa regido por un conjunto de algoritmos que de acuerdo a alguna ley física, biológica, económica, sociológica, etc., o de acuerdo con un modelo matemático, ejecutan en la pantalla transformaciones sobre representaciones simbólicas de una situación experimental. Es así como las simulaciones permiten actuar sobre sistemas cuya manipulación real resultaría imposible o muy costosa.

\section{Los ambientes lúdicos e interactivos}

En los últimos 15 años ha surgido una oleada de novedosos programas computacionales que posibilitan el acceso al conocimiento de manera ágil, dinámica y motivante: hipertextos, multimedia, hipermedia y la realidad virtual.

En este conjunto de programas también se deben ubicar los juegos, que han mostrado ser un ambiente excelente de aprendizaje por su poder seductor y capacidad didáctica de adaptarse al comportamiento del jugador y de esa manera ayudarlo a mejorar una habilidad o un proceso intelectual o motor. Conviene, entonces, clarificar cada uno de es tos conceptos.

\section{Hipertexto}

A diferencia de un texto 'plano' o lineal, la información contenida en un hipertexto se presenta de manera no secuencial, es decir, la información se organiza en forma de red, en cuyos nodos se almacena información gráfica y/o textual, de tal manera que el usuario, o lector del hipertexto, tiene la posibilidad de 'navegar' por la información a través de los nodos que conforman la red de acuerdo a sus 
intereses. Es decir, si un nodo en particular está conectado (encadenado), por ejemplo con otros cuatro nodos, el usuario tiene la posibilidad de ir a cualquiera de ellos, rom piendo así el orden lineal del texto plano.

Así, el hipertexto responde a la naturaleza del pensamiento humano, que es netamente asociativa. Por ejemplo, cuando pensamos en un 'perro', no sólo pensamos en el concepto 'perro', sino que imaginamos al perro en un contexto específico y con ciertos atributos: color, raza, tamaño, etc. El perro en el que pensamos podría llevarnos a recordar una situación en particular, y esta última conducirnos a otra diferente, y así sucesivamente. Como vemos, la asociación de conceptos juega un papel primordial en la naturaleza de la memoria (de corto y largo plazo).

El origen primero del hipertexto, en el contexto de la informática, se le concede a la propuesta de Vannevar Bush. Es él quien propone, hacia 1945, un dispositivo imaginario que denominó MEMOREX, fundamentado en un principio explicativo a cerca de como piensa el ser humano. Quien acuñó la palabra HIPERTEXTO fue Theodoro $\mathrm{H}$. Nelson en los años sesenta para significar con ella la escritura no lineal.

Propiedades del hipertexto

- El sistema está conformado por nodos de información encadenados entre si, formando una red.

- El sistema se puede expandir de tal manera que los usuarios puedan incluir sus propias ideas.

- Introduce estrategias de organización de archivos, que pemiten el manejo masivo de información.

- El hipertexto crea múltiples vías (estructuras, campos o alternativas) para que los lectores con diferentes intereses puedan decidir su propia secuencia de lectura y requerimientos particulares de información.

- Con el hipertexto, los lectores no están res tringidos a seguir la estructura de la materia en cuestión o la lógica de la secuencia con que el autor concibió el tema. De acuerdo con Nelson, cada estructura de conocimiento en cada sujeto es única, basada tanto en experiencias, capacidades únicas, como en formas particulares de acceso, interacción e interrelación con el conocimiento. La integración de nueva información a la estructura del conocimiento es un proceso individual. En consecuencia, es el texto quien debe acomodarse al lector y no el lector al texto. El hipertexto permite así, hacer más personal y más significativa la lectura.

\section{Multimedia}


El significado de multimedia ha sido utilizado con amplitud. Para algunos autores es un punto de confluencia (computador) de varias técnicas que permiten diferentes formas de representar conocimiento o información; para otros, es toda la metodología que permite una conexión lógica de los diferentes medios disponibles para obtener y almacenar toda una variedad de información, y por último, para otros, es el logro tecnológico de la electrónica reflejado en el alto poder de los computadores de hoy. Sin embargo, la idea fundamental es la integración lógica ya sea física o conceptual de todo tipo de representación y de transmisión del conocimiento humano.

Los ambientes multimediales implican la combinación de texto, gráficos, diagramas, animaciones (en $2 \mathrm{~d}$ y $3 \mathrm{~d}$ ), sonidos, e imágenes de video estáticas 0 animadas. El multimedia permite entonces un acceso a la información mediante el estímulo de los sentidos de la vista, el tacto y el oído.

Los conceptos que subyacen al multimedia están interrelacionados. Por ejemplo, las características de hardware y software disponibles para la producción de multimedia influyen en la calidad, cantidad y coherencia de los distintos archivos (gráficos, de video, audio y de animación) que se piensan incorporar en el producto multimedial.

Pero más que una mezcla de medios, multimedia es una interacción de lenguajes. Su diseño, por lo tanto, requiere de una comprensión de la autonomía de los lenguajes y sus posibilidades de utilización integral.

La tecnología multimedia se presenta amarrada a distintos recursos electrónicos, como el CD-ROM, WORM, disco óptico, tablas digitalizadoras, sintetizador, cámara de video, unidad de edición de video, video-disco, etc.

\section{Hypermedia}

Cuando el multimedia se conecta lógicamente formando un paquete interactivo, se le denomina hipemedia. En este caso, se almacenan los datos en nodos multimediales conectados por enlaces que a su vez son manipulados por un editor o un browser. El hipemedio posibilita la exploración de objetos mediante un encadenamiento no lineal, permitiendo al usuario navegar e interactuar con la información (utilizando para ello el ratón, el teclado o tocando la pantalla -touch screen-), es tableciendo búsquedas en el sentido del plano (en una misma pantalla a través de los objetos creados de video, texto, audio, etc.) o en el sentido de profundidad de campo (una página conduce a otra; un concepto permite profundizar y llegar a nuevos conceptos, o de un stack a otro, etc.), aprovechando para ello la naturaleza asociativa propia de la mente humana.

Un hipemedio, por lo tanto, debe servir para pres entar estructuras organizadas de la información, para representar conceptos y relaciones entre conceptos y para 
establecer sistemas de captura, recuperación y despliegue de información mediante objetos ( Trejo, 1996).

\section{Realidad virtual}

Son varias las definiciones que se han elaborado acerca de lo que es realidad virtual (VR), unas más sofisticadas que otras. Entre las más comunes están:

* VR es la experiencia de telepresencia, donde telepresencia es la sensación de presencia utilizando un medio de comunicación.

* VR es un modelo matemático que describe un "espacio tridimensional", dentro del cual están contenidos objetos que pueden representar cualquier cosa, desde una simple entidad geométrica, por ejemplo un cubo o una esfera, hasta una forma sumamente compleja como puede ser un desarrollo arquitectónico, un nuevo estado físico de la materia ó el modelo de una estructura de DNA.

* VR es un paso mas allá de lo que sería la simulación por computadora, tratándose mas bien de una simulación interactiva, dinámica y en tiempo real de un sistema.

* "Realidad Virtual es una manera mediante la cual los humanos visualizan, manipulan e interactúan con computadoras y datos extremadamente complejos" (David Blatner y Ste ve Auskatalnis )".

- Podemos clasificar en cuatro grandes grupos los sistemas que se proclaman como realidad virtual, ellos son:

- Sistemas Desktop de VR, VR en segunda persona, Sistemas de telepresencia y Sistemas de inmersión de VR.

\section{Desktop VR}

Engloban aquellas aplicaciones que muestran una imagen $2 \mathrm{D} \circ 3 \mathrm{D}$ en una pantalla de computador. Puesto que representan mundos de 3 dimensiones, los exploradores pueden viajar en cualquiera dirección dentro de estos mundos. Los ejemplos característicos de estos ambientes son los simuladores de vuelo para computadora o la mayoría de los juegos de alto nivel de realismo para computadora. 
VR en segunda persona.

Los sistemas en segunda persona (o unencumbered systems) involucran percepciones y respuestas en tiempo real a las acciones de los humanos involucrados, quienes están liberados o no están sometidos al uso de cascos, guantes, HMD's, alambres o cualquier otro tipo de interface "intrusiva". En los sistemas en segunda persona, el explorador sabe que está dentro del mundo virtual porque se ve a sí mismo dentro de la escena. Es decir, es un integrante del mundo virtual.

Sistemas de telepresencia

Los sistemas de telepresencia forman el cuarto grupo de aplicaciones de realidad virtual, los elementos que utilizan generalmente son cámaras, micrófonos, dispositivos táctiles y de fuerza con elementos de retroalimentación, ligados a elementos de control remoto para permitir al usuario manipular robots 0 dispositivos ubicados en localidades remotas mientras experimenta lo que experimentaría en el sitio en cuestión (pero de manera virtual).

Sistemas de inmersión

Son aquellos que sumergen o meten al explorador en el mundo virtual que estén tratando, mediante la utilización de sistemas visuales del tipo HMD, equipos seguidores de gestos y movimientos, así como elementos procesadores de sonido. De esta manera el participante queda estrechamente relacionado con el ambiente virtual, y aislado, hasta cierto punto, del mundo "real".

\section{Recursos en Internet}

Internet, sistema hipermedial por excelencia, abre las puertas a un nuevo mundo infinitamente rico en posibilidades educativas. A pesar de que en sus inicios tuvo un carácter puramente militarista, pues nace en la década del 60 de la Advance Research Project del Departamento de Defensa de Estados Unidos, hoy por hoy la red de redes pertenece a cualquier usuario que tenga la posibilidad de acceso a ella, lo que la ha hecho merecedora de calificativos como democratizante, globalizadora, interactiva y muchos otros que dan cuenta de todas las posibilidades académicas y socio-culturales que ofrece, en términos de acceso al conocimiento, eliminación de barreras espacio-temporales, contactos con diversas lenguas y culturas, interacción con usuarios de otras latitudes, entre otros. 
Dentro de este mar de posibilidades cabe destacar, en el ámbito educativo la presencia de los llamados cursos en linea (on line), programas desarrollados con fines explícitos de formación académica y manejado con diversidad de metodologías, que van desde el simple intercambio de archivos en red, has ta la interacción a través de listas de discusión, e-mails o chats, que derivan muchas veces en las llamadas aulas virtuales.

Igualmente, cobran relevancia los laboratorios virtuales como ambientes en los que es posible simular diversos fenómenos o procesos, en los que el usuario tiene la posibilidad de introducir modificaciones, probar hipótesis, inducir comportamientos.

Esto para mencionar solo una muestra de las ayudas educativas de punta, posibles gracias a los desarrollos teleinformáticos.

\section{B. Posibilidades pedagógicas que ofrecen las tecnologías de la información y la comunicación.}

La incursión abrupta de la informática al ámbito educativo no dio lugar a reflexiones previas desde la pedagogía, éstas han sido posteriores o paralelas, digamos que se ha establecido un diálogo entre la pedagogía y las áreas del conocimiento afines a ella(comunicación, filosofía) y la tecnología, a partir del cual se ha ido entretejiendo un rico y sólido constructo teórico como que ha permitido identificar las posibilidades pedagógicas que ofrece la incorporación de las tecnologías de la información y la comunicación como ayudas educativas en cualquier área del saber y que aquí se presentan desde tres perspectivas: desde la ciencia cognitiva y desde las ciencias el lenguaje. En adelante se usará el término HIPERMEDIA como concepto globalizante de las diferentes modalidades de ambientes tecnológicos antes descritos, ya que, potencialmente, incluye a las demás.

\section{Desde la Ciencia Cognitiva}

La Ciencia Cognitiva surge como un empeño contemporáneo de base empírica por responder a interrogantes epistemológicos de antigua data, en particular los vinculados a la naturaleza del conocimiento, sus elementos componentes, sus fuentes, evolución y difusión (Gardner, 1988). Es así como propone una visión holística del fenómeno de la cognición en el ser humano, al abordarlo desde las perspectivas de diferentes áreas del saber: antropología, filosofía, lingüística, neurociencia, informática, psicología cognitiva, Inteligencia Artificial, (Reber, 1985). Este trabajo interdisciplinario le ha permitido avanzar en la comprensión de muchos de los procesos relacionados con la cognición y son estos aportes los que, en las últimas décadas, han hecho evolucionar vertiginosamente, entre otros, el concepto sobre aprendizaje. 
Desde sus inicios, la ciencia cognitiva ha estado fuertemente relacionada con las ciencias de la computación, puesto que de una u otra manera ambas están interesadas en el funcionamiento de la mente humana, por lo que, además, ha existido una constante retroalimentación entre estas dos áreas del saber.

Es así como, por ejemplo, se encuentra una estrecha entre las características de los ambientes tecnológicos hipemediales y los procesos que, desde la ciencia cognitiva, se han planteado como relevantes en la adquisición del conocimiento, con base en lo cual es posible afirmar que los ambientes hipermediales enriquecen los procesos de aprendizaje y por tanto, hacen más efectiva tal adquisición. A continuación se presentan los principales argumentos que fundamentan es ta afirmación

a. La educación involucra procesos comunicacionales en los cuales la información, los mecanismos y los procesos para su transmisión se convierten en elementos relevantes. La calidad de la educación, entonces, debe estar fuertemente determinada por los conceptos, estrategias, métodos y medios que sean usados para manejar la información. La comunicación humana se fundamenta básicamente en la percepción a través de nuestros sentidos, de tal manera que para que se lleve a cabo eficientemente, la información debe también ser presentada "naturalmente", en forma multisensorial, lo cual es posible llevar a la práctica utilizando la tecnología multimedia que permite mostrar impresionantes imágenes de gran colorido y excelente resolución, incorporar animación y video en tiempo real y capturar sonido de alta calidad, todo lo cual incrementa la retención de información.

b. Los hipermedia, se basa en redes conceptuales, donde las relaciones significativas entre conceptos se establecen en forma de proposiciones, (dos conceptos unidos con palabras forman unidades semánticas). Aunque una red conceptual sea jerárquica, tiene la cualidad de permitir al usuario encontrar los conceptos básicos y las relaciones conceptuales de una forma libre, estableciendo una búsqueda lógica de los conceptos de manera autónoma. En los sistemas hipermediales la información se dispone en nodos que forman complejas redes, que permiten al usuario tener acceso a la información en forma no secuencial. En efecto, este tipo de estructura se asemeja más a la foma de pensamiento y conocimiento humanos, que son procesos básicamente asociativo, estructurados en mapas cognitivos (Término acuñado por E.C. Tolman) que están muy alejados de un secuenciamiento lineal y en estrecha relación con las experiencias individuales. Por esta razón, un proceso de aprendizaje será más exitoso en la medida en que los nuevos conceptos se vinculen a conceptos previos y se incorporen de esta manera al mapa cognitivo del individuo (Linn, 1996).

c. En los casos en que el usuario presenta dificultades de expresión o comprensión mediante la escritura alfabética, los hipemedia ofrecen 
una variedad de lenguajes, lo cual puede facilitar su proceso de aprendizaje. Esta concepción va de la mano con el planteamiento hecho por Gardner (1986) acerca de los diferentes tipos de inteligencias : el individuo posee inteligencias múltiples (lingüística, espacial, matemática, musical), cuyo desarrollo no se da unifomemente, por el contrario, dependiendo de su entomo cultural, de su mundo vivencial y, en muchos casos, de hechos fisiológicos, un tipo de inteligencia alcanza mayores niveles de desarrollo que los otros. Este factor es decisivo en los procesos de aprendizaje ya que el individuo tendrá un mejor desempeño en las áreas del saber relacionadas con la inteligencia en la que haya logrado un mayor desarrollo (nivel). Además, este hecho explica la relación entre representación simbólica y adquisición del conocimiento, en la medida en que ésta se facilita si la representación simbólica utilizada pertenece al campo de acción de la inteligencia más desarrollada. De ahí que el acceso al conocimiento por parte de un individuo cuya inteligencia más desarrollada sea la inteligencia lingüística, deberá darse, preferencialmente, a través del código verbal, muy al contrario de quien tenga más desarollada la inteligencia pictórica, en cuyo caso el acceso al conocimiento se facilitará si se apoya en códigos visuales.

d. Los hipermedia son un recurso adecuado para el autoaprendizaje (de hecho se basa más en el aprendizaje que en la enseñanza.) En este sentido, la posibilidad pedagógica que ofrece es permitir que el usuario organice y establezca las interrelaciones necesarias con la información, con el fin de aumentar o modificar su conocimiento previo. Este hecho está en estrecha relación con el planteamiento de los diferentes estilos de aprendizaje (Entwis tle, 1979) que se basa primordialmente en que no todos los sujetos acceden al conocimiento de la misma manera, por el contrario, cada individuo utiliza diferentes estrategias y ritmos de aprendizaje, lo cual está plenamente favorecido por las características de los ambientes hipermediales que permiten al usuario la posibilidad de suspendar la actividad en el momento que él quiere o que sus posibilidades de conocer, seleccionar o almacenar información se lo permiten; de igual forma le permite seleccionar, ordenar los conocimientos, representarlos y referirlos a otros, ejercitarse en los procesos de autoevaluación, comprobando, mediante ejercicios, su nivel de adquisición de conocimientos. Por otra parte, el estudiante no tiene la necesidad de encontrarse en las mismas coordenadas espaciotemporales que sus compañeros o sus docentes. En este mismo sentido, se amplía la cobertura de la educación.

e. El uso y producción de ambientes hipermediales favorece la autoreflexión y la toma de conciencia de la manera como cada usuario aprende, en la medida en que es constructor de su propio derrotero de aprendizaje y/o partícipe activo en la producción de nuevos hipemedia. Es decir, favorece la metacognición, entendida como conciencia de los propios recursos cognitivos con que cuenta el aprendiz (M. Esteban, 
1999), conciencia que involucra: 1) conocimiento de los objetivos que se quieren alcanzar con el esfuerzo mental; 2) elección de estrategias para conseguirlo; 3) auto-observación de la ejecución para comprobar si las estrategias elegidas son las adecuadas; 4) evaluación de los resultados para saber hasta qué punto se han logrado los objetivos (Burón, 1996).

Así, en la medida en que el individuo adquiere mayor consciencia de sus procesos metacognitivos, se incrementa su éxito en la adquisición de conocimiento. $Y$ en efecto, esa toma de conciencia se posibilita al hacer uso o al producir materiales hipermediales por cuanto se ve abocado a realizar funciones tales como ordenar, jerarquizar, agrupar el conocimiento y, además, otras necesarias para la adquisición de nuevos conocimientos como son la codificación de la información, a través de diversos procesos de selección, abstracción, interpretación o integración, y la recuperación de la misma (M. Esteban, 1999).

f. Una de las principales caracterís ticas de los entornos hipemediales es la interactividad (Bartolomé, 1995), es decir la posibilidad que ofrece al usuario de ser activo, de generar información intercambiando los roles de emisor y receptor, lo cual ya los aleja bastante de los llamados medios masivos de comunicación en los que unos pocos emisores "bombardean" con infomación a una masa estandarizada, pasiva y dispersa.

Además, en virtud de esta mis ma característica, el hipermedio se puede constituir en un micromundo en el que el usuario es un descubridor, puede probar hipótesis, transformar el entorno, identificar su lógica de funcionamiento mediante la exploración y la heur ística, todo lo cual, aplicado a ambientes educativos lo convierten en el protagon ista del proceso enseñanza-aprendizaje.

g. Por su capacidad para establecer redes de colaboración, el hipermedio facilita la interacción de los usuarios, aumentando las posibilidades de trabajo coorporativo y colaborativo y sus consecuentes beneficios para el aprendizaje (Salinas, 1995). Ya veíamos cómo Internet, hipermedia por excelencia, ofrece la posibilidad de que seres humanos con intereses comunes interactúen e intercambien información. Es así como en estos nuevos escenarios, profesores y estudiantes comparten los recursos materiales e informáticos de que disponen; los profesores aprenden al tiempo que los alumnos actualizando los conocimientos de su disciplina y proponiendo nuevas alternativas en el campo pedagógico, generando comunidades virtuales inteligentes, pues, en realidad, más allá de la conexión entre artefactos, asistimos a la interconexión de inteligencias.

h. El multimedia propicia la aproximación lúdica al conocimiento, por cuanto permite desarrollar simulaciones de eventos de la vida real, tutoriales, juegos, libros electrónicos, enciclopedias, y cualquier otro tipo de material de consulta, pero teniendo como perspectiva de desarrollo el entretenimiento y la persuasión. 
i. Por último, para potenciar al máximo estas posibilidades que ofrece el hipermedio como ambiente de aprendizaje, debe ser conjugado con técnicas de Inteligencia Artificial pues to que éstas permiten generar un mayor nivel de interactividad, reflejado por ejem plo, en la capacidad que presente el programa para modelar al estudiante, para hacer el seguimiento de su razonamiento y as i, en un momento dado, explicarle dónde estuvo el error, para ofrecerle diversas estrategias de acceso al conocimiento, para hacer simulaciones en las que el estudiante pueda poner a prueba hipótesis, etc. Todos estos aspectos fortalecerían la característica $\mathrm{m} s$ novedosa: que el producto se adecua al usuario y no como ha sido lo tradicional que el us uario se adecua al producto.

De esta forma, es posible concluir que las características de las tecnologías de la información y la comunicación, vistas a través de los entornos hipemediales, posibilitan un aprendizaje significativo, que según corrientes pedagógicas contemporáneas como la ciencia cognitiva y el constructivismo, tienen los siguientes rasgos (De Corte, 1997):

El aprendizaje es acumulativo:

Está basado en lo que los aprendices ya saben y pueden hacer, y en que pueden seleccionar y procesar activamente la información que encuentran. Consecuencia: cons truyen nuevo significado y desarrollan nuevas habilidades (De Corte, 1996).

El aprendizaje es autoregulado :

El aprendiz monitorea su propio proceso de construcción de conocimiento y adquisición de habilidades. Dependen cada vez menos de apoyo instruccional externo.

Se dirige a alcanzar metas, formuladas y autodeterminadas por el aprendiz de una manera explícita.

Es colaborativo: No es simplemente un proceso mental, requiere apoyo de otras actividades, interacciones con el contexto social y cultural, con actividades prácticas, con herramientas, con recursos.

Es individualmente diferente: Pues depende de motivaciones, conceptos previos, estilos cognitivos, estrategias individuales.

\section{Perspectiva literaria: nuevos lectores y nuevos escritores para la narrativa electrónica}

La creación literaria, siempre ansiosa de nuevos juegos del lenguaje y recursos narrativos encuentra hoy en los nuevos ambientes posibles gracias a las TIC, un 
vas to mundo por explorar y explotar. El sueño de algunos escritores (Cervantes, Joyce, Borges y Cortazar, el más osado, en este sentido, con su Rayuela) que se revelaban contra la linealidad a que los sometía el impreso, encuentra hoy en los hipemedia una vía de realización, que a mi modo de ver, supera lo soñado. Y nada más jus to que retribuirle a la literatura de alguna manera, pues to que fue de ella de donde surgieron realmente las primeras aproximaciones al hipertexto bajo la forma de la intra e intertextualidad, desde la creación literaria, y desde la teoría literaria, ya R. Barthes (Citado por Landow; 1995) en S/Z presenta un ideal de textualidad: "En este texto ideal abundan las redes (rés eaux) que actúan entre sí sin que ninguna pueda imponerse a las demás...; no tiene principio pero sí diversas vías de acceso, sin que ninguna de ellas pueda calificarse como principal; los códigos que moviliza se extienden hasta donde alcance la vista; son indeterminables...; los sistemas de significados pueden imponerse a este texto absolutamente plural, pero su número nunca está limitado ya que está basado en la infinidad del lenguaje" ${ }^{1}$ Esta puede considerarse como una definición muy adecuada de lo que es un hipertexto.

Landow, pionero en esta aventura de llamada narrativa electrónica (1985), muestra las bondades de este diálogo entre la tecnología y la literatura, específicamente la teoría crítica literaria contemporánea, por cuanto ella "promete teorizar el hipertexto, mientras que este promete encamar y, así, demostrar varios aspectos de la teoría, sobre todo los relativos a textualidad, narrativa y a los papeles o funciones de lector y es critor". 2

La posibilidad real de transfomar el final de una his toria, de recrearla con diversos lenguajes (video, sonido, texto, gráficas), de agregar, mutar o eliminar personajes, de re-elaborar la superestructura de un texto, de relacionar datos, de crear notas, no puede menos que enriquecer el hecho literario tanto desde el punto de vis ta del escritor como del lector, quienes ya no están más separados, ahora comparten sus roles, los alteman, juntos construyen sentido, ya sea a través de un texto abierto, de una creación colectiva, de una historia interactiva, o simplemente a través de la lectura hipertextual de un texto clásico.

De cualquier manera, todas estas posibles acciones implican modelos de lectura y escritura que conllevan, a su vez, "nuevas formas de representación, modos diferentes de sentir de ver y de decir" ${ }^{3}$ Formas de representar que tal vez responden de manera más adecuada a la cosmovisión de las nuevas generaciones, por cuanto están muy cercanas al lenguaje audiovisual del cine y la televisión, redescubriendo, por ejemplo, la imagen como imaginario comunicativo, después de tantos siglos de hegemonía del código lingüístico (la palabra); o

\footnotetext{
${ }^{1}$ En Landow, G Hipertexto-La convergencia de la teoría crítica contemporánea y la tecnología. Paidós, 1995, pag. 15.

${ }^{2}$ Landow, G. Hipertexto-La convergencia de la teoría crítica contemporánea y la tecnología. Paidós, 1995, pag. 14.
}

${ }^{3}$ Peña, B. L. Bernardo, De Melquíades a Microsoft. En: Memorias del Primer Congreso Nacional de Pedagogía, Gimnasio Moderno, oct. 1999, pág. 55. 
trasladando al texto (bajo la forma del hipermedio) una sintaxis con rupturas o superposiciones espacio-temporales, con acciones en paralelo y una polifonía de voces que genera un lector también polivalente capaz de "navegar" por multiplicidad de lenguajes y entornos sin ninguna aprehensión.

\section{Perspectiva lingüística}

Parafraseando a Saussure, cuando proponía una definición del signo lingüístico (Saussure 1916), podemos decir que leer y escribir son dos caras de una misma moneda: en efecto, se implican mutuamente, a pesar de lo cual cada una de estas actividades mantiene su propia caracterización. Es así como la lectura exige procesos de decodificación, que no son necesariamente los mismos que exige la escritura, pero en un orden contrario, como podría pensarse. La metáfora de la moneda nos permite comprender que, de cualquier forma, no podemos desligar estos dos hechos, por lo tanto, si abordamos el asunto de la escritura, estaremos abordando implícitamente el de la lectura y viceversa, como ocurre en las reflexiones que se plantean en los siguientes párrafos.

En la actualidad, a pesar de que subsisten vestigios del sentido elitista que tuvo la escritura durante siglos, el número de personas con acceso al código es crito se ha ampliado os tensiblemente, incluso hasta alcanzar, en los países desarrollados, el total de la población. Esto significa que en las diferentes sociedades, inclusive en la nuestra, el número de escritores potenciales se ha incrementado considerablemente. Sin embargo, son pocos los individuos que exploran y desarrollan su competencia escritora, lo cual no resulta demasiado trascendente en una sociedad en la que prime la cultura oral, no así, en una sociedad como la que enfrentamos hoy: la sociedad de la información, en la que más que nunca, leer y escribir se constituyen en actos vitales para un individuo que necesita y /o desea desenvolverse con éxito en los diferentes roles de su diario quehacer. En efecto, cada vez es mayor el número de lugares de trabajo, de recreación, de formación e inclusive de vivienda, cuyos procesos giran en torno a prácticas de lectura y escritura mediatizadas por el computador. La diversidad de herramientas que, en este sentido, ofrecen las tecnologías de la información y la comunicación evidencian la necesidad de que sus usuarios posean competencias lectoras y escritoras que, además posibilitar el manejo adecuado de estrategias ya conocidas, incluyan el desarrollo de habilidades que pemitan responder eficazmente a las exigencias de los nuevos entomos comunicativos y de interacción con el conocimiento.

Dichos entornos, ofrecen una gama de posibilidades para interactuar con la información, que va desde el correo electrónico has ta sistemas más elaborados, como los ambientes hipertextuales e hipermediales, pasando por una serie de 
herramientas que permiten una interacción más directa, como el Intemet Relay Chat y el "talk". 5 .

El correo electrónico, el "chat" y el "talk" pueden ser usados para codificar tanto escritos informales como fomales, con la diferencia de que el primero, por establecer una comunicación en diferido, da la posibilidad de revisar y re-elaborar el discurso, si se considera necesario. Por el contrario, las otras dos herramientas exigen un lecto-escritor más hábil, con mayor capacidad de respues ta inmediata y de precisión en el mensaje, por cuanto la interacción que se da a través de ellos es en tiempo real. Aquí el usuario corre el riesgo de ser desconocido deliberadamente si no alcanza las exigencias impuestas en la "conversación", en términos de una comprensión rápida y efectiva, de una escritura fluida y clara y, por supuesto, del grado de riqueza de los contenidos. Este último aspecto es el más relativo, ya que está en dependencia directa del tipo de contexto comunicativo. De hecho, existen ciberespacios informales en los que el código utilizado es muy cercano al oral, llegando indusive a crear signos para reemplazar elementos supralingüísticos (como la entonación) o paralingüísticos (como la gestualidad) como los que se muestran a continuación:

: ) "cara feliz"

:( "cara triste"

;) "picar el ojo"

:* "pico"

:o "admiración"

$\rightarrow \rightarrow @$ "una rosa"

En cuanto a los hipertextos, herramienta por excelencia para manejar información en los nuevos sis temas computacionales, bien sea en páginas web, programas en línea (on line) o paquetes multimediales exigen nuevas estrategias, nuevos compromisos y habilidades tanto en los lectores como en los escritores.

Estos nuevos escenarios de interacción generados por las TICs se constituyen, entonces, en nuevos escenarios comunicacionales cuyas caracteristicas los constituyen en ambientes propicios tanto para el estudio de diversos fenómenos lingüísticos como para la potencialización y/o desarrollo de habilidades comunicativas.

Queda de esta manera abierta la invitación a maestros y estudiantes para aproximarse a los ambientes de aprendizaje hipermediales, bien sea como usuarios o como creadores, redimencionarlos de acuerdo a sus propias necesidades e intereses y darles el sentido de verdaderos medios revilitalizadores

4 El IRC (htemet Relay Chat) es un servicio que le permite a varias personas interacuarsimultáneamente entre sí, de forma escrita a través de diversos canales creados en la red.

5 El "Talk" es un sistemade conferencia en tiempo real que permite a dos personæa manteneruna conversación. 
e innovadores del quehacer pedagógico y del proceso de adquisición de conocimiento.

\section{BIBLIOGRAFIA}

ADELL, Jordi. Tendencias en la educación en la sociedad de las tecnologías de la información. En: EDUDEC, Revista electrónica de tecnología educativa, $\mathrm{N}^{\circ} 7$, noviembre de 1997.

BARKLEY, Chris tine. Englis $h$ com puter critical thinking reading and writing interactive multimedia programs for comparison/contrast and analysis. Palomar Coll, San Marcos, California, 1994.

BLANDOW, D. Tools for overcoming thought barriers. Erfurt, Alemania,1989.

BROWN, Edward; Chignell Mark. Learning by linking: pedagogical environmnets for hypermedia authoring. En : joumal of computer in higher education, Vol. $5 \mathrm{~N}^{\circ} 1$, 1993.

BRUNER, Jerome. Realidad mental y mundos posibles. Gedisa, Barcelona, 1988. Actos de significado, Alianza, 1990.

BURÓN, J.(1996): Enseñar a aprender: introducción a la metacognición. Bilbao, Ediciones Mensajero, pp.15-27)

CANTOS, G. Pascual. El hipertextoen la enseñanza d elas lenguas asistida por ordenador. En: INFODIDAC, N 16, 1991.

COLOM ,C Hacia nuevos paradigmas educativos .Revista Studio Pedagógica, Salamanca 1984.

COOVERT, Robert. Findinf your way in hypertext: a guide to the software. En New York Times Book Review, junio de 1992.

DE CORTE Erik. Aprendizaje apoyado en el computador: Una perspectiva a partir de la investigación acerca del aprendizaje y la instrucción. Conferencia en el III Congreso Iberoamericano de Informática Educativa, RIBIE, Barranquilla, 1996 (Memorias).

DE SAUSSURE, Ferdinand. Curso de Lingüística General. Alianza, 1980.

DONALD G. Perrin en "La Universidad Electrónica del Futuro" Revista E.D. U.S. Distance Learning Ass ociation. Califomia, 1995. 
ESTEBAN, Manuel. Las estrategias de aprendizaje en el entorno de la Educación a Distancia $(E a D)$. Consideraciones para la reflexión y el debate. Universidad de Murcia, 1999.

GARDNER H. La nueva ciencia de la mente. Barcelona. Editorial Paidós Ingraf, 1988.

Inteligencias múltiples: la teoría en la práctica, Paidós, 1995

Mentes creativas: una anatomía de la creatividad, Paidós, 1995.

Mente no escolarizada: cómo piens an los niños, Paidós, 1995

Goel V. y Pirolli P. Structure of Design Problem Spaces., Cognitive Science, 1992.

HENAO Al varez Octavio. Efectos del uso de un procesador de textos y gráficos en el desarrollo de habilidades de escritura en niños de sexto grado. Informática Educativa, Vol.5 N.1,1992.

HUFFMAN, Donald; Goldberg,John.Using word processing to teach EFL composition. Journal of Computer-Based Instruction, vol. 20, N. 2,1993.

JONASSEN,David H.,Wang, Sherwood.Acquining Structural knowledge from semantically structured Hypertext. Journal of Computer-Based instruction.Winter 1993, Vol 20 \# 1,1-8.

LANDOW, George P. Hipertexto. La convergencia entre la teoría crítica contemporánea y la tecnología. Paidós, Barcelona, 1995.

LEON O., M. Idalith. Redes conceptuales en un proyecto multimedial. $\underline{\text { www.umb.edu.co1997 }}$

MAULE, William. Cognitive maps, Al agents and personalize virtual environments in Internet learning. En : Internet research, Intemetwork networking Aplications and Policy, Vol 8 N| 4, 1997.

NOVACK J.D. Aprendiencdo a aprender. Martínez Roca. Barcelona, 1988.

PANZA M. Opción critica en la didáctica . Revista Perfiles educativos № 186.

PAPAGIANNIS, G. et al. Information technology and education: implications for theory, practice and res earch. Ottawa:Intemational Developmental Research Centre, IDRC-MR161e. 1987.

PEÑA, L. Bernardo. De Melquíades a Microsoft. Sobre la difícil e inevitable relación entre tecnología y educación. Conferencia para el Primer _congreso Nacional de Pedagogía, Santafé de Bogotá, octubre de 1999 (Memorías). 
PORLAN, Rafael. Constructivismo y escuela, 1994.

PROVOST, Jyvie /1995). Planificación anual temática a partir de la Pedagogía del proyecto. Sherbrooke. Canadá.

RODRÍGUEZ, Gabriel (comp). La era teleinformática. Folios, México, 1985 Smith M. Curriculum models for the effective delivery of Information Technology capability in Secondary Schools. En Computer Education. York: NCC, 1992.

Sastre G ,M. moreno . Descubrimiento y construcción de conocimientos. De GEDISA, Barcelona, 1996.

SCHAFF, Adam. Lenguaje y conocimiento, Enlace-grijalbo, México, 1984

VAN DIJK, T. Estructuras y funciones del discurso. México, Siglo XXI, 1980. Texto y conte xto. Madrid, Cátedra, 1988.

VEGA, Manuel. Introducción a la psicología cognitiva, Alianza, 1994.

WERTSCH J.V. Vygotsk. La formación social de la mente. Barcelona, Editorial Paidós, 1988. 